\title{
Manejo higiénico en el servicio de alimentos: Codex Alimentarius y Normas Oficiales Mexicanas
}

\section{E. Ortega - Ibarraa¹ - A. Hernández - Jiménez²}

\begin{abstract}
RESUMEN
El propósito fundamental del manejo higiénico óptimo en los servicios de alimentación es el de disminuir la incidencia de Enfermedades Transmitidas por Alimentos (ETAS) y mejorar nuestros mecanismos de prevención, control y diagnóstico ante la inseguridad alimentaria-nutricional y aunado a esto a la biodisponibilidad de los nutrientes ingeridos en establecimientos públicos y privados. El perfil químico-médico-biológico y la adición de un fuerte componente como el nutricional, hace que la observación de los riesgos sanitarios sea fundamental para garantizar seguridad en cualquier espacio y no ser un problema de Salud Pública; con el fin de asegurar la disponibilidad, accesibilidad, aceptabilidad y la utilización biológica alimentaria. Específicamente es obligatorio corroborar el adecuado ambiente para la recepción de alimentos, almacenamiento, manejo de sustancias, refrigeración, congelación, áreas de cocción, preparación y montaje del alimento, servicios sanitarios, manejo de residuos, control de plagas entre otros con el único fin de asegurar una inocuidad alimentaria.
\end{abstract}

\begin{abstract}
The fundamental purpose of optimal hygienic management in food services is to reduce the incidence of foodborne diseases and improve our mechanisms for prevention, control and diagnosis. Optimal hygienic management is essential due to food-nutrition insecurity and, in addition, to the bioavailability of nutrients ingested in public and private establishments. The chemical-medical-biological profile and the addition of a strong component that needs to be incorporated as the nutritional one, makes the observation of health risks fundamental. It is important to provide a secure food environment to guarantee safety in any space and not create a problem in the arena of Public Health. There are several regulation points to meet and verify to continue providing a service in any place that has a food service. To establish an optimal hygienic food environment one must ensure availability, accessibility, acceptability and biological food use. Specifically, it is mandatory to assure an adequate environment for the reception of food, storage, handling of substances, refrigeration, freezing, cooking areas, preparation and plating, sanitary services, waste management and pest control among others. The sole purpose of providing these actions is for ensuring food safety.
\end{abstract}

Palabras Clave: seguridad alimentaria, nutrición, servicios de alimentación, codex alimentarius, normas oficiales mexicanas.

Keywords: food security, nutrition, food services, codex alimentarius, official Mexican standards.

\footnotetext{
1 Coordinador General del Centro de Investigación en Nutrición y Alimentación (CINA) y Director de la Licenciatura en Nutrición. Universidad del Istmo (UNISTMO) campus Juchitán de Zaragoza, Oaxaca. Profesor Investigador. eoi@bizendaa.unistmo.edu.mx

2 Estudiante de Licenciatura en Nutrición y asistente de investigación del Centro de Investigación en Nutrición y Alimentación (CINA) de la Universidad del Istmo (UNISTMO) campus Juchitán de Zaragoza, Oaxaca.

Contacto: Centro de Investigación en Nutrición y Alimentación (CINA) de la Licenciatura en Nutrición. Universidad del Istmo (UNISTMO) campus Juchitán de Zaragoza, Oaxaca. Carretera Transístmica Juchitán - La Ventosa Km. 14, La Ventosa, Oax., México C.P. 70102
} 
Acrónimos:

FAO Organización de las Naciones Unidas para la Agricultura y la Alimentación

OMS Organización Mundial de la Salud

SAN Seguridad Alimentaria y Nutricional

NOM Norma Oficial Mexicana

NMX Norma Mexicana

\section{Introducción}

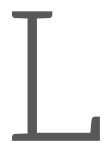

a seguridad alimentaria y nutricional (SAN) va ampliamente relacionada con la higiene e inocuidad de los alimentos lo cual sigue siendo un gran reto para los países subdesarrollados o en vías de desarrollo, debido a la morbilidad y mortalidad asociadas al consumo de alimentos contaminados química y biológicamente. Durante décadas la medición de la inocuidad de los alimentos se ha basado principalmente en la calidad microbiológica considerando dos aspectos fundamentales y críticos dentro del ámbito: la calidad del agua utilizada para el riego de alimentos y las prácticas de higiene e inocuidad adoptadas por los manipuladores de alimentos a lo largo de la cadena de producción (Baluka, 2015).

Garantizar la calidad y preservación de los alimentos se ha convertido en la principal obligación de todos los actores involucrados en la producción y comercialización de los mismos, tomando en cuenta que debe existir una organización, infraestructura y capacitación que cumplan con las necesidades de manera globalizada. De acuerdo con la literatura consultada, gran parte de los casos de las enfermedades alimentarias descritas en varios países son ocasionadas por el consumo de alimentos frescos y perecederos vendidos en mercados informales, la distribución de vegetales regados con agua contaminada como vía de transmisión de microorganismos (materia fecal, vía potencial para la dispersión de bacterias) y por la falta de saneamiento de los alimentos antes de ser consumidos. Es importante destacar que los riegos potenciales que ocasionan los alimentos contaminados tendrán mayor impacto en los grupos más vulnerables, como los infantes y adultos mayores (Uyttendaele, 2016).

La OMS/FAO: piedra angular para la inocuidad e higiene de los alimentos a nivel mundial

La normalización es un conjunto de actividades que tiene por objeto establecer especificaciones para los productos, procesos y servicios, así como la manera en que deben ser evaluadas. Dentro de la normalización se generan reglamentaciones de las características y la calidad de los productos con el fin de facilitar su producción y comercialización de manera que estos sean confiables y seguros al consumidor. La legislación sobre alimentos apareció en diversos países para prevenir la venta de productos fraudulentos, preocupándose inicialmente por los efectos adversos en la composición corporal. Actualmente se han extendido a otros aspectos de Salud Pública considerándose los más importantes la contaminación química, física y biológica (Calvo, 2012).

Desde el siglo XX surgió la necesidad de establecer normas alimentarias internacionales, una de las primeras organizaciones que intentó disponer de tales normas o estándares fue la Federación Internacional de Lechería, dicha institución creó aquellos lineamientos que podrían aplicarse a la leche y derivados. Fue en el año de 1956 cuando la Organización para la Alimentación y la Agricultura (FAO) y la Organización Mundial de la Salud (OMS) unieron sus esfuerzos para trabajar en materia de higiene e inocuidad de los alimentos a nivel mundial (Calvo, 2012).

Desde entonces dichos organismos contribuyen de manera responsable al proceso normativo aportando asesoramiento con fundamento científico, con la constante actualización de información en materia de inocuidad de los alimentos para evitar peligros de contaminación física, química, biológica y los relacionados con las nuevos procesos y tecnologías alimentarias durante la cadena de producción de alimentos (FAO, 2015).

En el año de 1963 se estableció el programa Codex Alimentarius bajo el control de la FAO y OMS con el propósito específico de alcanzar 
acuerdos internacionales sobre normas alimentarias y códigos de prácticas que pudieran salvaguardar la salud de los consumidores y estimular las buenas prácticas comerciales de manera globalizada. Los principios generales del Codex Alimentarius enmarcan la identificación de las prácticas esenciales involucradas en la higiene de los alimentos a lo largo de la cadena alimentaria comprendidas desde la producción primaria hasta el consumidor final, con el objeto de brindar alimentos aptos y fomentar la aplicación de esos principios, además de facilitar la orientación sobre códigos que puedan necesitarse en los procesos involucrados y productos básicos que permitan ampliar los requisitos de higiene específicos e implicados en cada uno de ellos (FAO, 2009).

Actualmente dichas normas comprenden aspectos relacionados con la composición y calidad de los alimentos distribuidos internacionalmente mediante métodos de análisis, el etiquetado de alimentos, códigos de prácticas para la reducción de riesgos de contaminación aplicables durante la producción, manipulación, distribución y transporte, evaluación de los alimentos modificados genéticamente, establecimiento de las dosis máximas de referencia para los aditivos, contaminantes y sustancias tóxicas que se presentan naturalmente en los alimentos, límites máximos de residuos para plaguicidas utilizados en la agricultura y medicamentos de uso veterinario (FAO,2015).

Estos principios generales establecen una base sólida para asegurar la higiene de los alimentos y deben aplicarse junto con cada código específico de prácticas de higiene cuando sea necesario y con las directrices sobre criterios microbiológicos. En este contexto, la FAO se esfuerza por atender y resguardar la seguridad alimentaria y nutricional sistemáticamente por medio de proyectos, programas, capacitación y publicaciones, además los gobiernos de cada país deben hacer una revisión al Codex y fomentar la aplicación de estos principios para proteger adecuadamente a la población de ETAS, asegurando que los alimentos que se ofrecen son de buena calidad y realizar programas de educación en la materia que permita comunicar de manera eficaz los principios de higiene a las industrias alimentarias (FAO et al, 2009).

Normalización de higiene e inocuidad de los alimentos en méxico

En México, la Ley Federal sobre Metrología y Normalización promueve la concurrencia de los sectores públicos, privados, científico y de consumidores, en la elaboración y observancia de Normas Oficiales Mexicanas (NOM) y Normas Mexicanas (NMX) teniendo como objetivo principal la unificación de criterios, facilitar la producción y comercialización de productos, homologar las normas mexicanas con las internacionales y definir la calidad y métodos de prueba para evitar actos desleales que impidan el incremento de la productividad y competitividad (Intedya, 2015).

El Sistema Federal de Salud en coordinación con la Comisión Federal para la Protección contra Riesgos Sanitarios (Cofepris) inició, consultó y decretó en diciembre del año 2009 la Norma Oficial Mexicana NOM-251-SSA1-2009: Prácticas de higiene para el proceso de alimentos, bebidas o suplementos alimenticios, la cual entró en vigor en septiembre de 2010. En dicha norma oficial se establecen los requisitos mínimos de buenas prácticas de higiene que deben observarse en el procesamiento de alimentos, bebidas, suplementos alimenticios y sus materias primas a fin de evitar su contaminación, y por lo tanto mejorar la transparencia y seguridad a lo largo de la cadena alimentaria (SSA, 2012).

Entre las buenas prácticas de manufactura contenida dentro de los párrafos de esta norma se encuentran disposiciones sobre instalaciones y áreas, equipos y utensilios, servicios, almacenamiento, control de operaciones y materias primas, prácticas de higiene personal, capacitación de los trabajadores, documentación, registros y protocolo para el retiro del producto cuando este sea un potencial peligro para los consumidores.

La NOM-251-SSA1-2009 puede ser aplicable a todas aquellas organizaciones encargadas del suministro alimentario y de manera obligatoria a las personas físicas o morales que se dedican 
al proceso de alimentos, bebidas y suplementos alimenticios dentro de territorio nacional mexicano, personal de plantas productoras de alimentos y bebidas, proveedores de insumos y servicios a la industria alimentaria, expendios de alimentos y personas interesadas en contar con las herramientas necesarias para la implementación de esta norma oficial (Intedya, 2015; SSA, 2010).

Es importante destacar que dentro delámbito privado se cuenta con la Sociedad Mexicana de Normalización y Certificación S.C NORMEX, el cual es una empresa que surgió el 3 de diciembre de 1993 encargada fundamentalmente en la elaboración de normas NMX-NORMEX, ofreciendo servicios en la certificación y verificación tras una evaluación extensa de productos, procesos y sistemas que garantice una excelente calidad en los servicios ofrecidos al consumidor. El estándar vigente dentro de la República Mexicana para la higiene e inocuidad de los alimentos específicamente en el ámbito de la hostelería y restauración es conocida como la NMX-F-605-NORMEX-2016: Manejo higiénico en el servicio de alimentos preparados para la obtención del Distintivo "H", con fecha de actualización y aprobación como Norma Mexicana el 7 de diciembre de 2015.

(NORMEX, 2016).

Dicha norma se publicó el 13 de octubre de 2004 en el Diario Oficial la Norma NMX-F605-NORMEX-2004 entrando en vigor el 12 de diciembre de 2004 para aplicarse a todos aquellos establecimientos de hostelería y restauración que de manera voluntaria desearan mejorar sus procesos para garantizar la seguridad e higiene de los alimentos incidiendo significativamente en la promoción turística de México ante el mundo (Intedya, 2015).

El Distintivo "H" es un elemento estratégico de la Secretaría de Turismo (SECTUR) y avalado por la Secretaria de Salud (SSA) para certificar a todos aquellos establecimientos prestadores de servicios de alimentos y bebidas que cumplan con los estrictos requisitos definidos dentro de la NMX-F-605-NORMEX- 2016 para disminuir la incidencia de las ETAS. La importancia de esta norma reside en promover y fomentar las buenas prácticas de higiene y sani- dad a lo largo de la cadena productiva primaria y secundaria, es decir, en la adecuada manipulación de los alimentos en el proceso de preparación y servicio considerando los aspectos "críticos" con el objeto de salvaguardar la salud de los consumidores (SECTUR, 2014).

En la actualidad se puede dar este reconocimiento a establecimientos fijos de alimentos y bebidas como: comedores industriales, cafeterías de hospitales, clubes deportivos, tiendas de autoservicio, entre otros que ofrecen el servicio de alimentación; siempre y cuando se cumpla con los estándares de higiene que marca la norma. Tiene una vigencia de un año y de no ser renovado, la empresa que lo ostente debe retirarlo de la vista del cliente (SECTUR, 2014). El impacto que tienen los elementos evaluadores es nacional e internacional y puntualiza la relevancia ante la búsqueda de evitar la vulnerabilidad a la inseguridad alimentaria y nutricional (ver Tabla 1).

Para otorgar la certificación del Distintivo "H" la SECTUR cuenta con un grupo de instructores distribuidos en todo el país especializados en el área químico-médica-biológica, de manera que cuando un establecimiento ha tomado la decisión de participar en el programa la SECTUR envía una lista con los nombres de los instructores a fin de que la entidad en cuestión seleccione el que sea de su agrado (NORMEX, 2016; SECTUR, 2014). A continuación, se menciona el proceso oficial para la obtención del Distintivo "H" (ver Tabla 2).

La obtención del Distintivo H se logra cuando se tienen los siguientes resultados:

- Contar como mínimo con el 80\% del personal operativo y el 100\% del personal de mandos medios capacitados en el curso que lleva por nombre "Manipulación Higiénica de los Alimentos" impartidos por los instructores registrados.

- Cumplir con los requisitos de higiene de los alimentos definidos en la NMX-F605-NORMEX-2016 detallados en la lista de verificación.

Para la realización del monitoreo se utiliza 
una lista de verificación que se encuentra dividida en dos estándares: los críticos que deberán cumplirse al 100\% y no críticos el 90\%. A continuación, se muestra los puntos contemplados en la evaluación:

- Recepción de alimentos

- Almacenamiento

- Manejo de sustancias químicas

- Refrigeración y congelación
- Área de cocina

- Preparación de alimentos

- Área de servicio

- Agua y hielo

- Servicios sanitarios para empleados

- Manejo de basura

- Control de plagas

- Personal

- Bar

Tabla 1. Comparación de objetivo, aplicación y nivel de impacto de los elementos evaluadores.

Elementos evaluadores.

Esta

Norma Oficial Mexicana NOM-251-SSA1-2009, "Prácticas de higiene para el proceso de alimentos, bebidas o suplementos alimenticios".

NMX - F - 605 - NOR MEX-2016 "Alimentos - manejo higiénico en el servicio de alimentos preparados para la obtención del Distintivo " $\mathrm{H}$ ".

Codex Alimentarius

\section{Objetivo}

Establecer requisitos mínimos de buenas prácticas de higiene que deben observarse en el procesamiento de alimentos, bebidas, suplementos alimenticios y sus materias primas a fin de evitar su contaminación, y por lo tanto mejorar la transparencia y seguridad a lo largo de la cadena alimentaria.

Promover y fomentar las buenas prácticas de higiene y sanidad a lo largo de la cadena productiva primaria y secundaria

\section{Garantizar alimentos} inocuos y de calidad a todas las personas y en cualquier lugar, bajo las normas alimentarias, directrices y códigos de prácticas internacionales.

\section{Aplicación}

Nivel de impacto

Todas aquellas organi- Nacional. zaciones encargadas del suministro alimentario y de manera obligatoria a las personas físicas o morales que se dedican al proceso de alimentos, bebidas y suplementos alimenticios dentro de territorio nacional mexicano.

Normas Oficiales Mexicanas (lineamientos de observancia obligatoria).

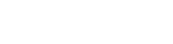

Todos aquellos estable- Nacional. cimientos de hostelería y restauración que de Expresan una recomenmanera voluntaria de- dación de parámetros o searan mejorar sus pro- procedimientos. cesos para garantizar la seguridad e higiene de Sí una NOM expresa la los alimentos incidiendo obligatoriedad de una significativamente en la NMX, ésta tendrá que promoción turística de cumplirse. México ante el mundo

Las normas y textos afi- Internacional. nes del Codex no sustituyen ni son una so- Normas alimentarias, lución alternativa a la directrices y códigos de legislación nacional. Las prácticas internacionaleyes y procedimientos les. administrativos de cada país contienen disposiciones que es necesario cumplir. 
Tabla 2. Pasos para la obtención del Distintivo "H"

\begin{tabular}{|l|l|}
\hline \multicolumn{1}{|c|}{ Elementos evaluadores. } & \multicolumn{1}{c|}{ Objetivo } \\
\hline Paso 1. Contratación del consultor "H" & $\begin{array}{l}\text { Selección del consultor "H" con credencial vigente por la SEC- } \\
\text { TUR. }\end{array}$ \\
\hline Paso 2. Diagnóstico & $\begin{array}{l}\text { El consultor "H" realiza pre-auditorias con la finalidad de de- } \\
\text { tectar puntos críticos, requerimientos y puntos de mejora que } \\
\text { permita la implementación del Sistema de Gestión "H" en las } \\
\text { áreas específicas de: recepción, almacenamiento, preparación y } \\
\text { servicio. }\end{array}$ \\
\hline Paso 3. Capacitación & $\begin{array}{l}\text { Capacitación en aula y campo impartido por el consultor a todo } \\
\text { el personal operativo, mandos medios y altos con el curso: "Ma- } \\
\text { nejo Higiénico de los Alimentos" con duración de 10 horas. }\end{array}$ \\
\hline Paso 4. Implementación & $\begin{array}{l}\text { Implementación del proceso de acuerdo a los lineamientos del } \\
\text { Sistema de Gestión "H" basándose en lo establecidos por la nor- } \\
\text { ma Mexicana vigente NMX-F-NORMEX-2016 y la lista de ve- } \\
\text { rificación. }\end{array}$ \\
\hline Paso 7. Emisión del Distintivo "H" & $\begin{array}{l}\text { Validación por parte del consultor que el establecimiento cum- } \\
\text { pla con lo necesario para posteriormente solicitar la verifica- } \\
\text { ción y obtener la certificación correspondiente. }\end{array}$ \\
\hline Paso 6. Verificación & $\begin{array}{l}\text { El establecimiento contrata a la Unidad de Verificación (acre- } \\
\text { ditadas por EMA-SECTUR) de acuerdo con sus necesidades, la } \\
\text { cual llevará acabo la visita de verificación final. }\end{array}$ \\
\hline cual emite de manera oficial el Distintivo "H".
\end{tabular}

Fuente: modificada y adaptado de SECTUR. (2014). Programa Manejo Higiénico de los Alimentos, Distintivo "H".

Conclusión

Los problemas de la Salud Pública bajo el enfoque de la alimentación y la nutrición dan a relucir complicaciones ante la búsqueda del óptimo bienestar personal y social, teniendo un complejo y nuevo término como el de la Seguridad Alimentaria y Nutricional para México. Es necesario tener la educación de la prevención y es tarea de cualquier sector involucrado y primordialmente ante los servicios de alimentación que tenemos a nuestro alcance. Teniendo en cuenta que la alimentación es una necesidad diaria, es difícil conocer la naturaleza del alimento e ignoramos aún más el proceso que cursa para tenerlo frente a nosotros antes de degustarlo. La normativa oficial nacional da el panorama conceptual y metodológico en busca de la prevención, manejo y control de la seguridad, por lo que es necesaria una actualización por parte de profesionales especializados en el tema que se trate, ya que así se mejoraría cualquier fin que se establezca en el documento.

Al conocer la literatura mínima requerida para mantener un lineamiento de sanidad, es necesario socializarlo en cualquier establecimiento que se dude de la calidad alimentaria y a su modo de preparación, siendo obligatorio 
para cualquier lugar que ofrezca un alimento con un almacenamiento inmediato, a mediano y largo plazo. Sustentar la problemática en la Salud Pública con la práctica errónea hará que sigamos con las mismas o nuevas complicaciones en los organismos; la solución existe en el conocimiento específico y a la generación de estrategias adecuadas para la reducción de alarmantes datos que no forman parte de una óptima inocuidad alimentaria.

\section{Referencias}

Baluka, S., Miller R., Kaneene J.B. (2015). Hygiene practices and food contamination in managed food service facilities in Uganda. En AfricanJournal of Food Science, 9(1), 31-42. Obtenido de http://www.cvm.msstate.edu/ images/academics/international/Baluka_et_al__-_Janaury 2015.pdf

Calvo, M.C. (2012). Toxicología de los alimentos. Ciudad de México, México: Mc Graw Hill.

FAO. (2015). Normas Internacionales y Códigos. Roma, Italia: FAO. Recuperado de http://www.fao.org/docrep/014/am859s/am859s14.pdf

FAO., OMS. (2009). Codex Alimentarius: Higiene de los Alimentos. Roma, Italia: FAO/OMS.

FAO/OMS. (2009). Informe de la Cuadragésima Primera Reunión del Comité del Codex Sobre Higiene de los Alimentos. Recuperado de http://www.fao.org/tempref/codex/ Reports/Alinorm10/al33_13s.pdf

Intedya.(2015).NORMA OFICIAL MEXICANA 251 México. Recuperado de http://www.intedya.com/internacional/92/consultoria-nom-251-mexico.html

Intedya. (2015). Distintivo H, estándares mexicanos de higiene para hostelería y restauración. Recuperado de http:// www.intedya.com/internacional/91/consultoria-distintivo-h-mexico.html

Normex. (2016). NMX-F-605-NORMEX-2016- Manejo Higiénico en el Servicio de Alimentos Preparados para la Obtención del Distintivo " $H$ ". Recuperado de https://www. gob.mx/cms/uploads/attachment/file/197511/NMX-F605-NORMEX-2016_ 7_de_diciembre_de_2015_firmada__002_.pdf

SSA. (2010). Norma Oficial Mexicana NOM-251SSA1-2009, Prácticas de higiene para el proceso de alimentos, bebidas o suplementos alimenticios. Recuperado de https://www.gob.mx/cms/uploads/attachment/file/138256/251ssa1.pdf
SECTUR. (2014).Programa Manejo Higiénico de los Alimentos, Distintivo " $H$ ". Recuperado de http://www. calidad.sectur.gob.mx/distintivoh/2014/10/17/programa-manejo-higienico-de-los-alimentos-distintivo-h/

Uyttendaele, M., Franz, E., Schlüter, O. (2016).Food Safety, a Global Challenge. En International Journal of Environmental Research and Public Health, 13(1), 67. doi: 10.3390/ijerph13010067 